\title{
CARD COUNTING IN CONTINUOUS TIME
}

\author{
PATRIK ANDERSSON,${ }^{*}$ Stockholm University
}

\begin{abstract}
We consider the problem of finding an optimal betting strategy for a house-banked casino card game that is played for several coups before reshuffling. The sampling without replacement makes it possible to take advantage of the changes in the expected value as the deck is depleted, making large bets when the game is advantageous. Using such a strategy, which is easy to implement, is known as card counting. We consider the case of a large number of decks, making an approximation to continuous time possible. A limit law of the return process is found and the optimal card counting strategy is derived. This continuous-time strategy is shown to be a natural analog of the discrete-time strategy where the so-called effects of removal are replaced by the infinitesimal generator of the card process.
\end{abstract}

Keywords: Sampling without replacement; invariance principle; gambling theory; optimal control

2010 Mathematics Subject Classification: Primary 60G40

Secondary $60 \mathrm{~F} 17$

\section{Introduction}

We will consider a typical house-banked casino card game, similar to, e.g. blackjack, trente et quarante, and baccarat, which is played for several coups before the deck is reshuffled. We thus have a case of sampling without replacement and as the game progresses the relative occurrence of the different cards will change and so will the expected value of the game. Card counting is the technique of using a betting strategy that takes advantage of these fluctuations in the expected value, making large bets when the game is advantageous and small bets when it is not. In addition, the betting strategy should be easy to use, i.e. be possible for the gambler to implement without the use of any aids. These ideas were first introduced in [10], where it was applied to blackjack.

Gottlieb [5] found an approximation of the expected return from card counting when using proportional betting. Equation (6.2) below, in the case of Kelly betting, can also be found there. Ekström and Wanntorp [2] considered a card game with two types of cards and derived a limit process. They then proceeded to find the optimal time to stop playing the game.

\section{A general card game}

The game is played with $D$ decks, each consisting of $c$ cards, all unique but not necessarily different with respect to the game, and we will assume that all permutations of the cards in the decks are equally likely. We let $C_{i}(i=1,2 \ldots, c D)$ denote the $i$ th card in the $D$ decks. The game is played for a number of coups without reshuffling and each coup requires exactly

Received 28 September 2010; revision received 20 September 2011.

* Postal address: Department of Mathematics, Stockholm University, 10691 Stockholm, Sweden.

Email address: patrika@math.su.se 
$m$ cards to determine the outcome. Many actual card games, like blackjack, require a stochastic number of cards to determine a coup. To avoid complications, we only treat the case of fixed $m$. We denote the profit (or loss) from betting one unit in coup $i$ by $Y_{i}=f\left(C_{m(i-1)+1}, \ldots, C_{i m}\right)$, where $f$ is some bounded, not necessarily symmetric, function. This function will depend on the rules of the game and the particular strategy used by the player. In general, the player's strategy, and thereby $f$, could vary as the deck is depleted, but we will assume a fixed $f$ for all coups. There is of course also a dependence on $D$ in $C_{i}$ and $Y_{i}$, but we sometimes suppress this in the notation for convenience. We will assume that the deck is reshuffled while there are still cards left, as is usually the case in actual casinos.

\section{Some general properties}

Some properties of the general card game will be useful. Let $\mathcal{F}_{i}=\sigma\left(C_{1}, \ldots, C_{m i}\right)$, i.e. the $\sigma$-algebra generated by the cards removed when the $i$ th coup is completed. Also, let $E_{i}=\mathrm{E}\left[Y_{i} \mid \mathcal{F}_{i-1}\right]$ be the expected value of a one unit bet in coup $i$ conditioned on the cards removed before the coup. Then $E_{i}$ is a martingale with respect to $\mathcal{F}_{i-1}$ and in particular $\mathrm{E}\left[E_{i}\right]=\mathrm{E}\left[Y_{1}\right]=E_{1}$ for all $i$. This fact is part of the so-called fundamental theorem of card counting, first introduced in [11] (see also [4]). Furthermore, it was shown in [4] that

$$
\begin{aligned}
E_{i} & =\mathrm{E}\left[f\left(C_{m(i-1)+1}, \ldots, C_{i m}\right) \mid \mathcal{F}_{i-1}\right] \\
& =\frac{(c D-m i) !}{(c D-m(i-1)) !} \sum_{j_{1}, \ldots, j_{m} \in\{m(i-1)+1, \ldots, c D\}} \mathrm{E}\left[f\left(C_{j_{1}}, \ldots, C_{j_{m}}\right) \mid \mathcal{F}_{i-1}\right] \\
& =\left(\begin{array}{c}
c D-m(i-1) \\
m
\end{array}\right) \sum_{m(i-1)+1 \leq j_{1}<\cdots<j_{m} \leq c D}^{-1} f^{\star}\left(C_{j_{1}}, \ldots, C_{j_{m}}\right),
\end{aligned}
$$

where $j_{1}, \ldots, j_{m}$ are distinct and

$$
f^{\star}\left(i_{1}, \ldots, i_{m}\right) \equiv \frac{1}{m !} \sum_{\pi \in S_{m}} f\left(\pi\left(i_{1}\right), \ldots, \pi\left(i_{m}\right)\right),
$$

with $S_{m}$ being the set of permutations of $\{1, \ldots, m\}$. The second equality follows since the $C_{i} \mathrm{~s}$ are exchangeable, and the third equality since the sum is symmetric in the remaining deck and, therefore, measurable with respect to $\mathcal{F}_{i-1}$. We may therefore conclude that $E_{i}$ is a $U$-statistic with symmetric kernel $f^{\star}$.

\section{Card counting in discrete time}

The idea of card counting is to, for each number of played coups $i$, assign each card a value, say $v_{i}\left(C_{j}\right)$, such that we can estimate the conditional expected value of the depleted deck by

$$
E_{i}\left(C_{1}, \ldots, C_{m(i-1)}\right)=\sum_{j=1}^{m(i-1)} v_{i}\left(C_{j}\right) .
$$

The best choice of $v_{i}$, in the sense of the least expected square error, i.e. the $E_{i}$ that minimizes

$$
\mathrm{E}\left[\left(\mathrm{E}\left[Y_{i} \mid C_{1}, \ldots, C_{m(i-1)}\right]-E_{i}\left(C_{1}, \ldots, C_{m(i-1)}\right)\right)^{2}\right]
$$


is

$$
\hat{E}_{i}\left(C_{1}, \ldots, C_{m(i-1)}\right)=E_{1}+\frac{c D-1}{c D-m(i-1)} \sum_{j=1}^{m(i-1)} e\left(C_{j}\right),
$$

where the quantities

$$
e(j)=\mathrm{E}\left[f\left(C_{2}, \ldots, C_{m+1}\right) \mid C_{1}=j\right]-E_{1}
$$

are known as the effects of removal. This result was first shown in [6] and can also be found in [3, pp. 415-417], where it was shown by solving the normal equations. It can also be viewed as a projection on a linear subspace of a $U$-statistic; see, e.g. [7, p. 459]. The gambler would keep adding the terms $(c D-1) e\left(C_{j}\right)$, thereby obtaining the so-called running count. When it is time to make a bet, the player divides the running count by the number of remaining cards in the deck, $c D-m(i-1)$, thereby obtaining the so-called true count. If this is a large number, the gambler expects the game to be favorable and makes a large bet. It should be noted that in practice the numbers $(c D-1) e\left(C_{j}\right)$ would be replaced by some correlated integers, thereby making the calculations easier to perform.

\section{Towards continuous time}

To study the problem in a continuous-time setting, we are interested in the cumulative profit $\sum_{i} Y_{D, i}$, properly scaled, as the number of decks becomes large. We will let $D=\infty$ indicate that the cards are sampled with replacement. In the following we let $W_{t}, W_{t}^{\circ}, \mathrm{d} W_{t}$, and $\mathrm{d} W_{t}^{\circ}$ denote Brownian motion, the Brownian bridge, and their respective differentials.

We note that since the cards are sampled without replacement, the different coups are dependent. The decks admit $\lfloor c D / m\rfloor$ coups and we thus define the total profit from betting one unit in a proportion $t \in[0,1)$ of the available coups as

$$
R_{D, t}=\sum_{i=1}^{\lfloor t c D / m\rfloor} Y_{D, i}
$$

Furthermore, note the decomposition

$$
\begin{aligned}
R_{D, t}-E_{D, 1}\left\lfloor t \frac{c D}{m}\right\rfloor= & \sum_{i=1}^{\lfloor t c D / m\rfloor}\left(Y_{D, i}-\mathrm{E}\left[Y_{D, i} \mid \mathcal{F}_{i-1}\right]\right) \\
& +\sum_{i=1}^{\lfloor t c D / m\rfloor}\left(\mathrm{E}\left[Y_{D, i} \mid \mathcal{F}_{i-1}\right]-E_{D, 1}\right)
\end{aligned}
$$

where $E_{D, 1}=\mathrm{E}\left[Y_{D, 1}\right]$.

As noted before, the deck is usually reshuffled before all cards have been used. We will therefore only consider the case $t<1$, thus avoiding technical problems.

The following definition and two lemmas will be useful.

Definition 5.1. (Martingale difference array.) Let $\left\{\xi_{n, i} ; i=1,2, \ldots, k_{n}\right\}$ be an array of random variables, and let $\left\{\mathcal{F}_{n, i} ; 0 \leq i \leq k_{n}\right\}$ be an array of sigma-algebras such that $\xi_{n, i}$ is $\mathcal{F}_{n, i}$-measurable and $\mathcal{F}_{n, i-1} \subset \mathcal{F}_{n, i}$. We then call $\xi_{n, i}$ a martingale difference array with respect to $\mathcal{F}_{n, i}$ if $\mathrm{E}\left[\xi_{n, i} \mid \mathcal{F}_{n, i-1}\right]=0$ almost surely for all $n$ and $i$. 
Lemma 5.1. ([8].) Suppose that $\xi_{n, i}$ is a martingale difference array and that $k_{n}(t)$ is a sequence of integer-valued, nondecreasing, right-continuous functions on $[0,1]$ such that $k_{n}(0) \equiv 0$ for all $n$. If

(i) $\max _{i \leq k_{n}(t)}\left|\xi_{n, i}\right| \stackrel{L_{2}}{\rightarrow} 0$, and

(ii) $\sum_{i=1}^{k_{n}(t)} \xi_{n, i}^{2} \stackrel{\mathrm{P}}{\rightarrow} t$ for each $t \in[0,1]$,

then $\sum_{i=1}^{k_{n}(t)} \xi_{n, i} \stackrel{J_{1}}{\Rightarrow} W_{t}$ as $n \rightarrow \infty$, i.e. we have weak convergence in Skorokhod's $J_{1}$ topology.

Lemma 5.2. ([1].) Let $\mathrm{P}_{\infty}$ and $\mathrm{P}_{n}$ be the probability measures representing sampling with and without replacement of $k$ balls from an urn containing $n$ balls with $c$ different types of balls, not necessarily equally represented. Then the total variation distance

$$
d_{\mathrm{TV}}\left(\mathrm{P}_{\infty}, \mathrm{P}_{n}\right) \leq \frac{2 c k}{n}
$$

We use this to show the following.

Lemma 5.3. It holds that

$$
\frac{1}{\sqrt{D}} \sum_{i=1}^{\lfloor t c D / m\rfloor}\left(Y_{D, i}-\mathrm{E}\left[Y_{D, i} \mid \mathcal{F}_{i-1}\right]\right) \stackrel{J_{1}}{\Rightarrow} \sigma_{1} W_{t} \quad \text { as } D \rightarrow \infty
$$

and

$$
\sigma_{1}^{2}=\frac{c}{m} \operatorname{var}\left(Y_{\infty, i}\right)
$$

Proof. We identify

$$
\xi_{D, i}=\frac{Y_{D, i}-\mathrm{E}\left[Y_{D, i} \mid \mathscr{F}_{i-1}\right]}{\sqrt{(c D / m) \mathrm{E}\left[\left(Y_{D, i}-\mathrm{E}\left[Y_{D, i} \mid \mathcal{F}_{i-1}\right]\right)^{2}\right]}}
$$

and $k_{D}(t)=\lfloor t c D / m\rfloor$. To see that the convergence of $\xi_{D, i}$ implies the lemma, we introduce the notation $c_{2}=\left(c_{m(i-1)+1}, \ldots, c_{m i}\right)$ and $\boldsymbol{C}_{1}=\left(C_{1}, \ldots, C_{m(i-1)}\right)$, and $\mathrm{P}_{\infty}$ and $\mathrm{P}_{D}$ as the probability measures representing sampling the cards from the $D$ decks with and without replacement, respectively. We then have

$$
\begin{aligned}
\left|\mathrm{E}\left[Y_{D, i} \mid \mathcal{F}_{i-1}\right]-\mathrm{E}\left[Y_{\infty, i}\right]\right| & =\left|\sum_{\boldsymbol{c}_{2}} f\left(\boldsymbol{c}_{2}\right) \mathrm{P}_{D}\left(\boldsymbol{c}_{2} \mid \boldsymbol{C}_{1}\right)-\sum_{\boldsymbol{c}_{2}} f\left(\boldsymbol{c}_{2}\right) \mathrm{P}_{\infty}\left(\boldsymbol{c}_{2}\right)\right| \\
& \leq f_{\max } \sum_{\boldsymbol{c}_{2}}\left|\mathrm{P}_{D}\left(\boldsymbol{c}_{2} \mid \boldsymbol{C}_{1}\right)-\mathrm{P}_{\infty}\left(\boldsymbol{c}_{2} \mid \boldsymbol{C}_{1}\right)\right| \\
& =O\left(\frac{1}{D}\right) \\
& \rightarrow 0 \quad \text { as } D \rightarrow \infty
\end{aligned}
$$

since the probability conditioned on the cards removed again represents sampling without replacement, only from a different urn. Analogously, we find that

$$
\lim _{D \rightarrow \infty} \mathrm{E}\left[Y_{D, i}\right]=\mathrm{E}\left[Y_{\infty, i}\right]
$$


We also have

$$
\begin{aligned}
\mathrm{E}\left[Y_{D, i} \mathrm{E}\left[Y_{D, i} \mid \mathcal{F}_{i-1}\right]\right] & =\sum_{\boldsymbol{c}_{1}, \boldsymbol{c}_{2}} \mathrm{P}_{D}\left(\boldsymbol{c}_{1}, \boldsymbol{c}_{2}\right) f\left(\boldsymbol{c}_{2}\right) \mathrm{E}\left[Y_{D, i} \mid \boldsymbol{c}_{1}=\boldsymbol{c}_{1}\right] \\
& \rightarrow \sum_{\boldsymbol{c}_{1}, \boldsymbol{c}_{2}} \mathrm{P}_{\infty}\left(\boldsymbol{c}_{1}, \boldsymbol{c}_{2}\right) f\left(\boldsymbol{c}_{2}\right) \mathrm{E}\left[Y_{\infty, i}\right] \\
& =\sum_{\boldsymbol{c}_{2}} \mathrm{P}_{\infty}\left(\boldsymbol{c}_{2}\right) f\left(\boldsymbol{c}_{2}\right) \mathrm{E}\left[Y_{\infty, i}\right] \\
& =\mathrm{E}\left[Y_{\infty, i}\right]^{2} \quad \text { as } D \rightarrow \infty
\end{aligned}
$$

So that

$$
\lim _{D \rightarrow \infty} \mathrm{E}\left[\left(Y_{D, i}-\mathrm{E}\left[Y_{D, i} \mid \mathcal{F}_{i-1}\right]\right)^{2}\right]=\operatorname{var}\left(Y_{\infty, i}\right) .
$$

Next we verify the conditions of Lemma 5.1. The first condition holds since $Y_{D, i}$ is bounded. Towards showing that the second condition holds, we note that

$$
\mathrm{E}\left[\sum_{i=1}^{\lfloor t c D / m\rfloor} \xi_{D, i}^{2}\right]=\sum_{i=1}^{\lfloor t c D / m\rfloor} \frac{m}{c D} \frac{\mathrm{E}\left[\left(Y_{D, i}-\mathrm{E}\left[Y_{D, i} \mid \mathcal{F}_{i-1}\right]\right)^{2}\right]}{\mathrm{E}\left[\left(Y_{D, i}-\mathrm{E}\left[Y_{D, i} \mid \mathcal{F}_{i-1}\right]\right)^{2}\right]} \rightarrow t \quad \text { as } D \rightarrow \infty
$$

Also,

$$
\begin{aligned}
\mathrm{E}[( & \left.\left.\sum_{i=1}^{\lfloor t c D / m\rfloor} \xi_{D, i}^{2}\right)^{2}\right] \\
= & \sum_{i=1}^{\lfloor t c D / m\rfloor} \sum_{j=1}^{\lfloor t c D / m\rfloor}\left(\frac{m}{c D}\right)^{2} \frac{\mathrm{E}\left[\left(Y_{D, i}-\mathrm{E}\left[Y_{D, i} \mid \mathcal{F}_{i-1}\right]\right)^{2}\left(Y_{D, j}-\mathrm{E}\left[Y_{D, j} \mid \mathcal{F}_{j-1}\right]\right)^{2}\right]}{\mathrm{E}\left[\left(Y_{D, i}-\mathrm{E}\left[Y_{D, i} \mid \mathcal{F}_{i-1}\right]\right)^{2}\right] \mathrm{E}\left[\left(Y_{D, j}-\mathrm{E}\left[Y_{D, j} \mid \mathcal{F}_{j-1}\right]^{2}\right]\right.} \\
= & \left\lfloor\frac{t c D}{m}\right\rfloor\left(\frac{m}{c D}\right)^{2} \frac{\mathrm{E}\left[\left(Y_{D, i}-\mathrm{E}\left[Y_{D, i} \mid \mathcal{F}_{i-1}\right]\right)^{4}\right]}{\mathrm{E}\left[\left(Y_{D, i}-\mathrm{E}\left[Y_{D, i} \mid \mathcal{F}_{i-1}\right]\right)^{2}\right]^{2}} \\
& +\left(\left\lfloor\frac{t c D}{m}\right\rfloor^{2}-\left\lfloor\frac{t c D}{m}\right\rfloor\right)\left(\frac{m}{c D}\right)^{2} \\
& \times \frac{\mathrm{E}\left[\left(Y_{D, i}-\mathrm{E}\left[Y_{D, i} \mid \mathcal{F}_{i-1}\right]\right)^{2}\left(Y_{D, j}-\mathrm{E}\left[Y_{D, j} \mid \mathcal{F}_{j-1}\right]\right)^{2}\right]}{\mathrm{E}\left[\left(Y_{D, i}-\mathrm{E}\left[Y_{D, i} \mid \mathcal{F}_{i-1}\right]\right)^{2}\right] \mathrm{E}\left[\left(Y_{D, j}-\mathrm{E}\left[Y_{D, j} \mid \mathcal{F}_{j-1}\right]\right)^{2}\right]} \\
\rightarrow & t^{2},
\end{aligned}
$$

by exchangeability and by again using Lemma 5.2. Thus,

$$
\operatorname{var}\left(\sum_{i=1}^{\lfloor t c D / m\rfloor} \xi_{D, i}^{2}\right) \rightarrow 0 \quad \text { as } D \rightarrow \infty
$$

which implies the second condition of Lemma 5.1.

Now, for the second term in decomposition (5.1), we will use the following result.

Lemma 5.4. ([9].) Let $U_{D, i}$ be a $U$-statistic with kernel $f^{\star}$ based on a sample of size $i$ taken without replacement from a population of size $N_{D}$, where $N_{D} \rightarrow \infty$ as $D \rightarrow \infty$. That is,

$$
U_{D, i}=\left(\begin{array}{c}
i \\
m
\end{array}\right)^{-1} \sum_{1 \leq i_{1}<\cdots<i_{m} \leq i} f^{\star}\left(C_{D, i_{1}}, \ldots, C_{D, i_{m}}\right) .
$$


Define $E_{D, 1}=\mathrm{E}\left[f^{\star}\left(C_{D, 1}, \ldots, C_{D, m}\right)\right]$ and $\tilde{E}_{D, 1}=\mathrm{E}\left[f^{\star}\left(C_{D, 1}, \ldots, C_{D, m}\right) \mid C_{D, 1}\right]$, and assume that

(i) $\lim _{D \rightarrow \infty} \mathrm{E}\left[\left(\tilde{E}_{D, 1}-E_{D, 1}\right)^{2}\right]=\sigma_{2}^{2}$,

(ii) $\sup _{D \in \mathbb{N}} \mathrm{E}\left[f^{\star}\left(C_{D, 1}, \ldots, C_{D, m}\right)^{2}\right]<\infty$,

(iii) $\sup _{D \in \mathbb{N}} \mathrm{E}\left[\left|\tilde{E}_{D, 1}-E_{D, 1}\right|^{2+\delta}\right]<\infty$ for some $\delta>0$.

Now let

$$
\zeta_{D, t}= \begin{cases}0 & \text { if } t<m / N_{D}, \\ \frac{\left\lfloor t N_{D}\right\rfloor\left(U_{D,\left\lfloor t N_{D}\right\rfloor}-E_{D, 1}\right)}{m \sqrt{N_{D} \sigma_{2}^{2}}} & \text { if } m / N_{D} \leq t \leq 1 .\end{cases}
$$

Then $\zeta_{D, t} \stackrel{J_{1}}{\Rightarrow} W_{t}^{\circ}$ as $D \rightarrow \infty$.

We now obtain the weak convergence of the second term in the decomposition.

Lemma 5.5. It holds that

$$
\frac{1}{\sqrt{D}} \sum_{i=1}^{\lfloor t c D / m\rfloor}\left(\mathrm{E}\left[Y_{D, i} \mid \mathcal{F}_{i-1}\right]-E_{D, 1}\right) \stackrel{J_{1}}{\Rightarrow} \sqrt{c} \sigma_{2} \int_{0}^{t} \frac{W_{s}^{\circ}}{1-s} \mathrm{~d} s, \quad t<1,
$$

where $\sigma_{2}^{2}=\operatorname{var}\left(\tilde{E}_{\infty, 1}\right)$.

Proof. Let $U_{D, i}=\mathrm{E}\left[f\left(C_{D, i+1}, \ldots, C_{D, i+m}\right) \mid C_{D, 1}, \ldots, C_{D, i}\right]$, i.e. the conditional expected value with $i$ cards removed. Then $U_{D, i}$ is a $U$-statistic and $E_{D, i}=U_{D, m(i-1)}$.

The second and third conditions of Lemma 5.4 follow since $f$ is bounded. The first condition can be shown by using Lemma 5.2, similarly to the proof of Lemma 5.3. Comparing (3.1) with Lemma 5.4 we see that in (3.1) the index refers to the number of coups not in the summation while in Lemma 5.4 it refers to the number that are in the summation. The indices thus run in opposite directions and we obtain

$$
\frac{(c D-\lfloor t c D\rfloor)\left(U_{D,\lfloor t c D\rfloor}-E_{D, 1}\right)}{m \sqrt{c D \sigma_{2}^{2}}} \stackrel{J_{1}}{\Rightarrow} W_{1-t}^{\circ} \stackrel{\mathrm{D}}{=} W_{t}^{\circ},
$$

where

$$
\sigma_{2}^{2}=\operatorname{var}\left(\tilde{E}_{\infty, 1}\right)
$$

We also note that

$$
Z_{D, t}=\frac{(c D-m\lfloor t c D / m\rfloor)\left(U_{D, m\lfloor t c D / m\rfloor}-E_{D, 1}\right)}{m \sqrt{c D \sigma_{2}^{2}}} \stackrel{J_{1}}{\Rightarrow} W_{t}^{\circ}
$$

for the same reasons. Now, for $t<1$,

$$
\begin{aligned}
& \frac{1}{\sqrt{D}} \sum_{i=1}^{\lfloor t c D / m\rfloor}\left(\mathrm{E}\left[Y_{D, i} \mid \mathcal{F}_{i-1}\right]-E_{D, 1}\right) \\
& =\frac{1}{\sqrt{D}} \sum_{i=0}^{\lfloor t c D / m\rfloor-1}\left(U_{D, m i}-E_{D, 1}\right) \\
& =\frac{1}{\sqrt{D}} \int_{0}^{\lfloor t c D / m\rfloor}\left(U_{D, m\lfloor s\rfloor}-E_{D, 1}\right) \mathrm{d} s
\end{aligned}
$$




$$
\begin{aligned}
& =\frac{1}{\sqrt{D}} \int_{0}^{\lfloor t c D / m\rfloor} Z_{D, s m / c D} \frac{m \sqrt{c D \sigma_{2}^{2}}}{c D-m\lfloor s\rfloor} \mathrm{d} s \\
& =\frac{1}{\sqrt{D}} \int_{0}^{\lfloor t c D / m\rfloor(m / c D)} Z_{D, s} \frac{m \sqrt{c D \sigma_{2}^{2}}}{c D-m\lfloor s c D / m\rfloor} \frac{c D}{m} \mathrm{~d} s \\
& \stackrel{J_{1}}{\Rightarrow} \sqrt{c} \sigma_{2} \int_{0}^{t} \frac{W_{s}^{\circ}}{1-s} \mathrm{~d} s,
\end{aligned}
$$

where the convergence follows by the continuous mapping theorem.

Theorem 5.1. The sequence

$$
\frac{R_{D, t}-\lfloor t c D / m\rfloor E_{D, 1}}{\sqrt{D}}, \quad t<1,
$$

is tight and each limit point (in the sense of weak convergence) is a continuous semimartingale with decomposition $R_{t}=M_{t}+A_{t}$, where

$$
M_{t} \stackrel{\mathrm{D}}{=} \sigma_{1} W_{t}
$$

and

$$
A_{t} \stackrel{\mathrm{D}}{=} \sqrt{c} \sigma_{2} \int_{0}^{t} \frac{W_{s}^{\circ}}{1-s} \mathrm{~d} s .
$$

Remark 5.1. Note that Theorem 5.1 does not specify the joint distribution of the decomposition and, thus, does not specify the distribution of $R_{t}$. However, knowing the marginal distributions in the decomposition will be enough for our purpose.

Proof of Theorem 5.1. Let $M_{D, t}$ denote the left-hand side of (5.2), and let $A_{D, t}$ denote the left-hand side of (5.3). Then it follows from Lemmas 5.3 and 5.5 that the sequence

$$
\left(\begin{array}{c}
M_{D, t} \\
A_{D, t}
\end{array}\right)
$$

is tight and that all limit points have the marginal distributions given in the theorem. Therefore, $M_{D, t}+A_{D, t}$ is tight and each limit point can be given the above decomposition.

\section{In continuous time}

Theorem 5.1 justifies the approximation of $R_{D, t}$ for large $D$ by the solution to the stochastic differential equation (SDE)

$$
\mathrm{d} R_{t}=\left(\sqrt{c D} \sigma_{2} \frac{W_{t}^{\circ}}{1-t}+\frac{c D}{m} E_{D, 1}\right) \mathrm{d} t+\sqrt{D} \sigma_{1} \mathrm{~d} W_{t} \equiv \mu_{t} \mathrm{~d} t+\sqrt{D} \sigma_{1} \mathrm{~d} W_{t},
$$

with $R_{0}=0$. In the continuation we treat the case of continuous time and take (6.1) as our model of a continuous-time card game. Note that we suppress the dependence on $D$ in the notation $\mathrm{d} R_{t}$ and $\mu_{t}$, and that the limiting values $\sigma_{1}$ and $\sigma_{2}$ can be replaced by the exact variances for a $D$-deck game. We assume that at every instant we bet a proportion $a_{t}$ of our current fortune $F_{t}$ so that our fortune evolves according to

$$
\mathrm{d} F_{t}=a_{t} F_{t} \mathrm{~d} R_{t}=a_{t} F_{t}\left(\mu_{t} \mathrm{~d} t+\sqrt{D} \sigma_{1} \mathrm{~d} W_{t}\right), \quad F_{0}=1,
$$


where $a_{t}$ is assumed to be an adapted semimartingale. Our objective is to choose $a_{t}$ in such a way as to maximize our fortune. The objective of maximizing the fortune is not well posed unless we quantify this further. The standard solution in gambling problems, which is also sometimes used in economics and finance, is to maximize the expected logarithmic fortune at some terminal time. In gambling theory this is usually referred to as Kelly's strategy and it turns out to have some desirable properties; see, e.g. [3]. We are therefore interested in the logarithm of our fortune, which according to Itô's lemma satisfies

$$
\begin{aligned}
\mathrm{d}\left(\log F_{t}\right) & =\frac{1}{F_{t}} \mathrm{~d} F_{t}-\frac{1}{2} \frac{1}{F_{t}^{2}} \mathrm{~d}[F, F]_{t} \\
& =a_{t}\left(\mu_{t} \mathrm{~d} t+\sqrt{D} \sigma_{1} \mathrm{~d} W_{t}\right)-\frac{1}{2} \frac{1}{F_{t}^{2}} a_{t}^{2} F_{t}^{2} D \sigma_{1}^{2} \mathrm{~d} t \\
& =\left(a_{t} \mu_{t}-\frac{1}{2} a_{t}^{2} D \sigma_{1}^{2}\right) \mathrm{d} t+a_{t} \sqrt{D} \sigma_{1} \mathrm{~d} W_{t}
\end{aligned}
$$

Assuming that $a_{t}$ is regular enough, we have

$$
\frac{\mathrm{d}}{\mathrm{d} t} \mathrm{E}\left[\log F_{t}\right]=\mathrm{E}\left[a_{t} \mu_{t}-\frac{1}{2} a_{t}^{2} D \sigma_{1}^{2}\right]=\frac{1}{2 D \sigma_{1}^{2}}\left(\mathrm{E}\left[\mu_{t}^{2}\right]-\mathrm{E}\left[\left(a_{t} D \sigma_{1}^{2}-\mu_{t}\right)^{2}\right]\right) .
$$

Now, $\mathrm{E}\left[\mu_{t}^{2}\right]$ is a parameter of the game which we cannot control and what then remains, in order to maximize $\mathrm{E}\left[\log F_{t}\right]$, is to minimize

$$
\int_{0}^{t} \mathrm{E}\left[\left(a_{s} D \sigma_{1}^{2}-\mu_{s}\right)^{2}\right] \mathrm{d} s .
$$

That is, we want to minimize the mean square error between the expected value of our game and our betting proportion multiplied by the variance and the number of decks.

\section{Betting strategies}

The continuation now depends on the restrictions we put on $a_{t}$. The most liberal restriction would be to let $a_{t} \in \mathcal{F}_{t}$ and this should also be the most favorable to the gambler. A more realistic restriction would be to instead let $a_{t}$ be a linear function of the removed cards. This is more realistic since in a practical situation this would be possible for the gambler to implement and this is the one we would consider to be card counting. Let us denote the optimal choice in the first case by $a_{t}^{*}$ and in the second case by $\hat{a}_{t}$.

\subsection{No restrictions}

Letting $a_{t} \in \mathcal{F}_{t}$, the optimal strategy is of course to choose

$$
a_{t}=\frac{\mu_{t}}{D \sigma_{1}^{2}} \equiv a_{t}^{*}
$$

Now, since

$$
\mu_{t}=\sqrt{c D} \sigma_{2} \frac{W_{t}^{\circ}}{1-t}+\frac{c D}{m} E_{D, 1} \sim \mathrm{N}\left(\frac{c D}{m} E_{D, 1}, \frac{c D \sigma_{2}^{2} t}{1-t}\right)
$$

we obtain

$$
\frac{\mathrm{d}}{\mathrm{d} t} \mathrm{E}\left[\log F_{t}\right]=\frac{\left(c D E_{D, 1} / m\right)^{2}}{2 D \sigma_{1}^{2}}+\frac{c \sigma_{2}^{2}}{2 \sigma_{1}^{2}} \frac{t}{1-t}
$$


and

$$
\mathrm{E}\left[\log F_{t}\right]=\frac{\left(c D E_{D, 1} / m\right)^{2}}{2 D \sigma_{1}^{2}} t-\frac{c \sigma_{2}^{2}}{2 \sigma_{1}^{2}}[t+\log (1-t)] .
$$

In most casino games it is not allowed to bet a negative amount; the optimal strategy then is to choose

$$
a_{t}=\max \left\{a_{t}^{*}, 0\right\}
$$

We then obtain

$$
\begin{aligned}
\frac{\mathrm{d}}{\mathrm{d} t} \mathrm{E}\left[\log F_{t}\right] & =\frac{1}{2 D \sigma_{1}^{2}} \mathrm{E}\left[\mu_{t}^{2}-\left(\max \left\{\mu_{t}, 0\right\}-\mu_{t}\right)^{2}\right] \\
& =\frac{1}{2 D \sigma_{1}^{2}} \mathrm{E}\left[\mathbf{1}_{\left\{\mu_{t}>0\right\}} \mu_{t}^{2}\right] \\
& =\frac{1}{2 D \sigma_{1}^{2}} \mathrm{E}\left[\mu_{t}^{2} \mid \mu_{t}>0\right] \mathrm{P}\left(\mu_{t}>0\right) .
\end{aligned}
$$

Now, since, for $\zeta \sim \mathrm{N}\left(\mu, \sigma^{2}\right)$, we have

$$
\mathrm{E}\left[\zeta^{2} \mid \zeta>0\right]=\sigma^{2}+\mu^{2}+\mu \sigma \frac{\phi(\mu / \sigma)}{\Phi(\mu / \sigma)}
$$

where $\phi$ and $\Phi$ are the standard normal density and distribution functions, respectively, we obtain

$$
\begin{aligned}
\frac{\mathrm{d}}{\mathrm{d} t} \mathrm{E}\left[\log F_{t}\right]=\frac{1}{2 D \sigma_{1}^{2}} & {\left[\left(\frac{c D \sigma_{2}^{2} t}{1-t}+\left(\frac{c D E_{D, 1}}{m}\right)^{2}\right) \Phi\left(\frac{\sqrt{c D} E_{D, 1}}{m \sigma_{2}} \sqrt{\frac{1-t}{t}}\right)\right.} \\
& \left.+\frac{(c D)^{3 / 2} \sigma_{2} E_{D, 1}}{m} \sqrt{\frac{t}{1-t}} \phi\left(\frac{\sqrt{c D} E_{D, 1}}{m \sigma_{2}} \sqrt{\frac{1-t}{t}}\right)\right] .
\end{aligned}
$$

In the special case where $E_{D, 1}=0$, which is approximately true in many casino games, we find that

$$
\mathrm{E}\left[\log F_{t}\right]=-\frac{c \sigma_{2}^{2}}{4 \sigma_{1}^{2}}[t+\log (1-t)],
$$

where we note that $t+\log (1-t)<0$ for $t>0$. We see that the expected logarithmic fortune of the player is independent of the number of decks. Since the time it takes to reach this fortune is proportional to the number of decks, this explains why the player should prefer to play a game with fewer decks.

\subsection{Linear function}

Now, let $a_{t}$ be a linear function of the removed cards. To this end, let $X_{D, t}$ be the $c$-dimensional vector of cards removed after a fraction $t$ of the available coups has been played. Denote the normalized limit of this process by $X_{t}$, i.e.

$$
\frac{X_{D, t}-i t D}{\sqrt{D}} \stackrel{J_{1}}{\Rightarrow} X_{t},
$$

where $i$ is a $c$-column vector of $1 \mathrm{~s}$. It follows from standard arguments that $X_{t}$ is a Gaussian 
process with mean 0 and variance of the $j$ th component determined by

$$
\begin{aligned}
\operatorname{var}\left(\frac{X_{D, t}^{(j)}-t D}{\sqrt{D}}\right) & =\frac{1}{D} \frac{\lfloor t c D / m\rfloor m D(c D-\lfloor t c D / m\rfloor m)(c D-D)}{(c D)^{2}(c D-1)} \\
& \rightarrow t(1-t) \frac{c-1}{c} \\
& =\operatorname{var}\left(X_{t}^{(j)}\right) \quad \text { as } D \rightarrow \infty,
\end{aligned}
$$

since $X_{D, t}^{(j)}$ is hypergeometrically distributed. Since the components of $X_{t}$ are exchangeable and the sum of the components is deterministic, we also obtain

$$
0=\operatorname{var}\left(\sum_{j=1}^{c} X_{t}^{(j)}\right)=c \operatorname{var}\left(X_{t}^{(j)}\right)+c(c-1) \operatorname{cov}\left(X_{t}^{(j)}, X_{t}^{(k)}\right),
$$

so that

$$
\operatorname{cov}\left(X_{t}^{(j)}, X_{t}^{(k)}\right)=-t(1-t) \frac{1}{c} \text { for } j \neq k
$$

We summarize these calculations by stating that

$$
\mathrm{E}\left[X_{t} X_{t}^{\top}\right]=t(1-t)\left(\mathbb{I}-\frac{1}{c} i i^{\top}\right)
$$

Based on the above we make the approximation that, for large $D, X_{D, t}$ is distributed as $i D t+\sqrt{D} X_{t}$.

We set $a_{t}=X_{D, t}^{\top} \beta_{t}$, where $\beta_{t}$ is a $c$-dimensional vector. We aim to determine the optimal $\beta_{t}, \hat{\beta}_{t}$, and, thus, $\hat{a}_{t} \equiv X_{D, t}^{\top} \hat{\beta}_{t}$. The drift $\mu_{t}$ of the SDE (6.1) can be thought of as a function $\mu\left(X_{D, t}\right)$. The optimal $\beta_{t}$ should then minimize

$$
\mathrm{E}\left[\left(D \sigma_{1}^{2} a_{t}-\mu_{t}\right)^{2}\right]=\mathrm{E}\left[\left(D \sigma_{1}^{2} X_{D, t}^{\top} \beta_{t}-\mu\left(X_{D, t}\right)\right)^{2}\right] .
$$

We differentiate with respect to $\beta_{t}$ to obtain

$$
\begin{aligned}
0 & =\frac{\partial}{\partial \beta_{t}} \mathrm{E}\left[\left(D \sigma_{1}^{2} X_{D, t}^{\top} \beta_{t}-\mu\left(X_{D, t}\right)\right)^{2}\right] \\
& =2 D^{2} \sigma_{1}^{4} \mathrm{E}\left[X_{D, t} X_{D, t}^{\top}\right] \beta_{t}-2 D \sigma_{1}^{2} \mathrm{E}\left[X_{D, t} \mu\left(X_{D, t}\right)\right] .
\end{aligned}
$$

Now, since $\mathrm{E}\left[X_{D, t} X_{D, t}^{\top}\right]=D^{2} t^{2} i i^{\top}+D \mathrm{E}\left[X_{t} X_{t}^{\top}\right], \hat{\beta}_{t}$ is determined by

$$
\sigma_{1}^{2} D^{2}\left(D t^{2} i i^{\top}+\mathrm{E}\left[X_{t} X_{t}^{\top}\right]\right) \hat{\beta}_{t}=\mathrm{E}\left[X_{D, t} \mu\left(X_{D, t}\right)\right] .
$$

Let $\bar{\beta}_{t}=\sum_{k} \hat{\beta}_{t}^{(k)}$. Then the $j$ th row of (7.5) now reads, using (7.3),

$$
\sigma_{1}^{2} D^{2}\left[D t^{2} \bar{\beta}_{t}+\frac{t(1-t)}{c}\left((c-1) \hat{\beta}_{t}^{(j)}-\left(\bar{\beta}_{t}-\hat{\beta}_{t}^{(j)}\right)\right)\right]=\mathrm{E}\left[X_{D, t}^{(j)} \mu\left(X_{D, t}\right)\right] .
$$

Summing these $c$ equations over $j$ we obtain, since $\sum_{j} X_{D, t}^{(j)} \equiv c D t$,

$$
\sigma_{1}^{2} D^{2}\left[c D t^{2} \bar{\beta}_{t}+\frac{t(1-t)}{c}\left((c-1) \bar{\beta}_{t}-\left(c \bar{\beta}_{t}-\bar{\beta}_{t}\right)\right)\right]=c D t \frac{c D}{m} E_{D, 1} .
$$


This implies that

$$
\bar{\beta}_{t}=\frac{c E_{D, 1}}{m D \sigma_{1}^{2} t}
$$

Solving for $\hat{\beta}_{t}^{(j)}$ now gives

$$
\hat{\beta}_{t}^{(j)}=\frac{\mathrm{E}\left[X_{D, t}^{(j)} \mu\left(X_{D, t}\right)\right]-D t c D E_{D, 1} / m}{D^{2} \sigma_{1}^{2} t(1-t)}+\frac{E_{D, 1}}{m D \sigma_{1}^{2} t} .
$$

It remains to calculate $\mathrm{E}\left[X_{D, t} \mu\left(X_{D, t}\right)\right]$. We know from the previous calculations that there are random variables $Z_{1}$ and $Z_{2}$, with zero-mean Gaussian marginal distributions independent of $t$, such that

$$
\left[\begin{array}{c}
X_{D, t}^{(j)} \\
\mu\left(X_{D, t}\right)
\end{array}\right] \stackrel{\mathrm{D}}{=}\left[\begin{array}{c}
D t+\sqrt{D t(1-t)} Z_{1} \\
c D E_{D, 1} / m+\sqrt{t(1-t)} Z_{2} /(1-t)
\end{array}\right] .
$$

We may then write

$$
\begin{aligned}
\mathrm{E}\left[X_{D, t}^{(j)} \mu\left(X_{D, t}\right)\right] & =\mathrm{E}\left[\left(D t+\sqrt{D t(1-t)} Z_{1}\right)\left(\frac{c D}{m} E_{D, 1}+\frac{\sqrt{t(1-t)}}{1-t} Z_{2}\right)\right] \\
& =t\left(\sqrt{D} \mathrm{E}\left[Z_{1} Z_{2}\right]+\frac{c D^{2} E_{D, 1}}{m}\right) ;
\end{aligned}
$$

the fact that $\mathrm{E}\left[Z_{1} Z_{2}\right]$ is not a function of $t$ can be motivated through a limit argument, so that $\mathrm{E}\left[X_{D, t} \mu\left(X_{D, t}\right)\right]$ is a linear function of $t$. In particular, we have

$$
\frac{\mathrm{E}\left[X_{D, t} \mu\left(X_{D, t}\right)\right]}{t}=\lim _{h \rightarrow 0} \frac{\mathrm{E}\left[X_{D, h} \mu\left(X_{D, h}\right)\right]}{h}=\left.\mathcal{A}(x \mu(x))\right|_{x=0} \equiv A_{0},
$$

where $\mathcal{A}$ is the infinitesimal generator of $X_{D, t}$. This, together with (7.6) and the definition of $\hat{a}_{t}$, gives

$$
\begin{aligned}
\hat{a}_{t} & =X_{D, t}^{\top} \hat{\beta}_{t} \\
& =X_{D, t}^{\top} \frac{A_{0} / D-i c D E_{D, 1} / m}{D \sigma_{1}^{2}(1-t)}+\frac{E_{D, 1}}{m D \sigma_{1}^{2} t} \sum_{i=1}^{c} X_{D, t}^{(i)} \\
& =\frac{1}{D \sigma_{1}^{2}}\left[\frac{c D E_{D, 1}}{m}+X_{D, t}^{\top} \frac{A_{0} / D-i c D E_{D, 1} / m}{1-t}\right] .
\end{aligned}
$$

Remark 7.1. Comparing the estimate of the conditional expectation given in the square brackets in (7.8) with (4.1) we see that both are the unconditional expectation plus a linear function of the removed cards divided by the fraction of cards remaining. In the continuoustime case the infinitesimal generator is the analog of the effects of removal from the discrete-time case.

Towards calculating the growth of the fortune, we find that

$$
\begin{aligned}
\frac{\mathrm{d}}{\mathrm{d} t} \mathrm{E}\left[\log F_{t}\right] & =\mathrm{E}\left[\hat{a}_{t} \mu_{t}\right]-\frac{1}{2} D \sigma_{1}^{2} \mathrm{E}\left[\left(\hat{a}_{t}\right)^{2}\right] \\
& =\mathrm{E}\left[\hat{\beta}_{t}^{\top} X_{D, t} \mu_{t}\right]-\frac{1}{2} D \sigma_{1}^{2} \mathrm{E}\left[\left(\hat{\beta}_{t}^{\top} X_{D, t}\right)^{2}\right] \\
& =\frac{1}{2} D \sigma_{1}^{2} \hat{\beta}_{t}^{\top} \mathrm{E}\left[X_{D, t} X_{D, t}^{\top}\right] \hat{\beta}_{t} \\
& =\frac{1}{2} D^{3} \sigma_{1}^{2} t^{2}\left(\bar{\beta}_{t}\right)^{2}+\frac{1}{2} D^{2} \sigma_{1}^{2} \hat{\beta}_{t}^{\top} \mathrm{E}\left[X_{t} X_{t}^{\top}\right] \hat{\beta}_{t},
\end{aligned}
$$


where the third equality uses (7.4). Calculations now show that

$$
\begin{aligned}
\hat{\beta}_{t}^{\top} \mathrm{E}\left[X_{t} X_{t}^{\top}\right] \hat{\beta}_{t} & =t(1-t) \sum_{j=1}^{c}\left(\hat{\beta}_{t}^{(j)}-\frac{\bar{\beta}_{t}}{c}\right)^{2} \\
& =t(1-t) \sum_{j=1}^{c}\left(\frac{A_{0}^{(j)}-D c D E_{D, 1} / m}{D^{2} \sigma_{1}^{2}(1-t)}\right)^{2} \\
& =\frac{t}{(1-t) D^{4} \sigma_{1}^{4}}\left(A_{0}^{2}-D^{2} c\left(\frac{c D}{m} E_{D, 1}\right)^{2}\right),
\end{aligned}
$$

where $A_{0}^{2} \equiv \sum_{j=1}^{c}\left(A_{0}^{j}\right)^{2}$. Then

$$
\frac{\mathrm{d}}{\mathrm{d} t} \mathrm{E}\left[\log F_{t}\right]=\frac{\left(c D E_{D, 1} / m\right)^{2}}{2 D \sigma_{1}^{2}}+\frac{t}{1-t} \frac{1}{2 D^{2} \sigma_{1}^{2}}\left(A_{0}^{2}-D^{2} c\left(\frac{c D}{m} E_{D, 1}\right)^{2}\right),
$$

and, thus,

$$
\mathrm{E}\left[\log F_{t}\right]=\frac{\left(c D E_{D, 1} / m\right)^{2}}{2 D \sigma_{1}^{2}} t-\frac{1}{2 D^{2} \sigma_{1}^{2}}\left(A_{0}^{2}-D^{2} c\left(\frac{c D}{m} E_{D, 1}\right)^{2}\right)(t+\log (1-t)) .
$$

We note that if

$$
\frac{A_{0}^{2}}{c D^{2}}=\sigma_{2}^{2}+\left(\frac{c D E_{D, 1}}{m}\right)^{2}
$$

i.e. if the variance of $a_{t}^{*}$ and $\hat{a}_{t}$ are equal and, thus, the variance of the residual from the linearization is $0,(7.1)$ and (7.9) are equal and the expected logarithmic fortune does not depend on which of the two restrictions we choose. In fact, straightforward calculations show the correlation between $a_{t}^{*}$ and $\hat{a}_{t}$ :

$$
\operatorname{corr}\left(a_{t}^{*}, \hat{a}_{t}\right)=\sqrt{\frac{A_{0}^{2}-c D^{2}\left(c D E_{D, 1} / m\right)^{2}}{c D^{2} \sigma_{2}^{2}}} .
$$

\section{Example}

We consider the following game, essentially the same as in Example 11.3.6 of [3], where we have a deck with two cards, one red and one black. Upon placing a one-unit wager, the player either makes a (net) profit of three units if two consecutive cards are red, or loses the one unit wagered. We thus have $c=m=2$ and

$$
Y_{i}= \begin{cases}3 & \text { if } C_{2 i-1}=C_{2 i}=\mathrm{red} \\ -1 & \text { otherwise. }\end{cases}
$$

Easy calculations show that in a $D$-deck game the expected value is

$$
E_{D, 1}=\mathrm{E}\left[Y_{D, i}\right]=-\frac{1}{2 D-1} \rightarrow 0 \quad \text { as } D \rightarrow \infty,
$$

so that the game is fair in the limit of many decks. We also have $\mathrm{E}\left[\tilde{E}_{\infty}\right]=0$ and

$$
\begin{aligned}
\sigma_{2}^{2} & =\mathrm{E}\left[\tilde{E}_{\infty}^{2}\right] \\
& =\mathrm{E}\left[\mathrm{E}\left[f^{\star}\left(C_{\infty, 1}, C_{\infty, 2}\right) \mid C_{\infty, 1}\right]^{2}\right] \\
& =\frac{1}{2} \mathrm{E}\left[f^{\star}\left(C_{\infty, 1}, C_{\infty, 2}\right)^{2} \mid C_{\infty, 1}=\mathrm{red}\right]+\frac{1}{2} \mathrm{E}_{\infty}\left[f^{\star}\left(C_{\infty, 1}, C_{\infty, 2}\right)^{2} \mid C_{\infty, 1}=\text { black }\right]
\end{aligned}
$$




$$
\begin{aligned}
& =\frac{1}{2}\left(3 \cdot \frac{1}{2}+(-1) \cdot \frac{1}{2}\right)^{2}+\frac{1}{2}(-1)^{2} \\
& =1 \\
\sigma_{1}^{2} & =\frac{c}{m} \operatorname{var}\left(Y_{\infty, i}\right)=\frac{c}{m} \mathrm{E}_{\infty}\left[Y_{\infty, i}\right]^{2}=\frac{2}{2}\left(3^{2} \cdot \frac{1}{4}+(-1)^{2} \cdot \frac{3}{4}\right)=3 .
\end{aligned}
$$

The limiting approximation of the return process of this game thus has the dynamics

$$
\mathrm{d} R_{t}=\left(\sqrt{2 D} \frac{W_{t}^{\circ}}{1-t}-\frac{D}{2 D-1}\right) \mathrm{d} t+\sqrt{3 D} \mathrm{~d} W_{t} .
$$

Let

$$
X_{D, t}=\left[\begin{array}{c}
X_{D, t}^{(1)} \\
X_{D, t}^{(2)}
\end{array}\right]=\left[\begin{array}{c}
\text { \#red cards removed } \\
\text { \#black cards removed }
\end{array}\right] .
$$

The optimal betting strategy involves $\mu_{t}$. In discrete time this corresponds to

$$
\frac{c D}{m} \mathrm{E}\left[Y_{D,\lfloor t c D / m\rfloor} \mid X_{D,\lfloor t c D / m\rfloor / D}\right],
$$

where the factor $c D / m$ is due to $t$ running from 0 to 1 , while the index of the discrete processes runs from 1 to $c D / m$. The optimal betting strategy is thus

$$
a_{t}^{*}=\frac{\mu_{t}}{D \sigma_{1}^{2}}=\frac{2 D}{2} \frac{\mathrm{E}\left[Y_{D,\lfloor t D\rfloor} \mid X_{D,\lfloor t D\rfloor / D}\right]}{3 D},
$$

where

$$
\begin{aligned}
\mathrm{E}\left[Y_{D,\lfloor t D\rfloor} \mid X_{D,\lfloor t D\rfloor / D}\right] & =4 \frac{\left(D-X_{D, t}^{(1)}\right)\left(D-X_{D, t}^{(1)}-1\right)}{\left(2 D-X_{D, t}^{(1)}-X_{D, t}^{(2)}\right)\left(2 D-X_{D, t}^{(1)}-X_{D, t}^{(2)}-1\right)}-1 \\
& =\frac{\left(1-X_{D, t}^{(1)} / D\right)\left(1-X_{D, t}^{(1)} / D-1 / D\right)}{(1-t)(1-t-1 /(2 D))}-1 .
\end{aligned}
$$

For large $D$, an approximation of the optimal betting proportion is thus given by

$$
a_{t}^{*} \approx \frac{\left(1-X_{D, t}^{(1)} / D\right)^{2}}{3(1-t)^{2}}-\frac{1}{3} .
$$

To find the optimal card counting strategy, we use (7.7) and choose $t=1 /(2 D)$, i.e. the time after one card has been removed. We find that

$$
\begin{aligned}
A_{0} & =\frac{\mathrm{E}\left[X_{D, t} \mu\left(X_{D, t}\right)\right]}{t} \\
& =2 D \mathrm{E}\left[X_{D, 1 /(2 D)} \mu\left(X_{D, 1 /(2 D)}\right)\right] \\
& =2 D \mathrm{E}\left[X_{D, 1 /(2 D)} \frac{2 D}{2} \mathrm{E}\left[Y \mid X_{D, 1 /(2 D)}\right]\right] .
\end{aligned}
$$

Easy calculations also show that

$$
\begin{aligned}
& \mathrm{E}\left[Y \mid X_{D, 1 /(2 D)}=\left[\begin{array}{l}
1 \\
0
\end{array}\right]\right]=-\frac{3}{2 D-1}, \\
& \mathrm{E}\left[Y \mid X_{D, 1 /(2 D)}=\left[\begin{array}{l}
0 \\
1
\end{array}\right]\right]=\frac{1}{2 D-1} .
\end{aligned}
$$




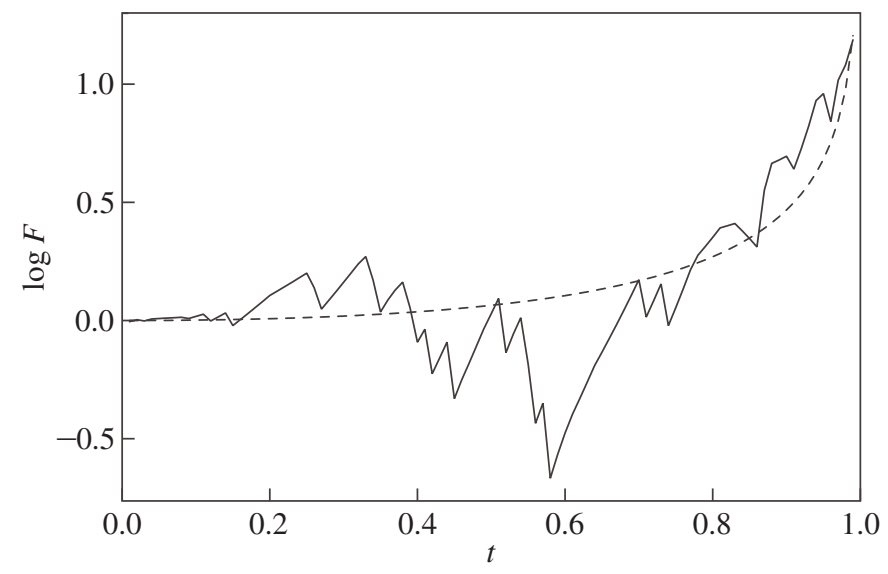

FIGURE 1: E $\left[\log F_{t}\right]$ (dashed line) and a simulated trajectory (solid line) for the game in the example with $D=100$.

We then obtain

$$
A_{0}=2 D^{2} \frac{1}{2}\left(\left[\begin{array}{l}
1 \\
0
\end{array}\right] \frac{-3}{2 D-1}+\left[\begin{array}{l}
0 \\
1
\end{array}\right] \frac{1}{2 D-1}\right)=\frac{D^{2}}{2 D-1}\left[\begin{array}{c}
-3 \\
1
\end{array}\right] .
$$

So that, according to (7.8), the optimal betting proportion in the linear case is, asymptotically as $D \rightarrow \infty$,

$$
\hat{a}_{t}=\frac{1}{3(2 D-1)}\left(-1+\frac{X_{D, t}^{\top}}{1-t}\left[\begin{array}{c}
-2 \\
2
\end{array}\right]\right) \approx \frac{X_{D, t}^{\top}}{3 D(1-t)}\left[\begin{array}{c}
-1 \\
1
\end{array}\right] .
$$

We also see that $\operatorname{corr}\left(a_{t}^{*}, \hat{a}_{t}\right) \rightarrow 1$, so the optimal betting proportion is asymptotically perfectly correlated with the betting proportion in the linear case and there is no loss in the expected logarithmic fortune in using a linear function.

With these strategies we are able to calculate $\mathrm{E}\left[\log F_{t}\right]$ using (7.2) or (7.10), which is plotted in Figure 1 together with a sample trajectory of $\log F_{t}$.

\section{Acknowledgements}

The author is grateful to an anonymous referee and to Andreas N. Lagerås for helpful comments. The author also thanks Stewart Ethier for providing comments on a version of this paper that appeared in the author's PhD thesis. Part of this research was conducted under the fellowship program JSPS Postdoctoral Fellowship for North American and European Researchers (Short Term).

\section{References}

[1] Diaconis, P. and Freedman, D. (1980). Finite exchangeable sequences. Ann. Prob. 8, 745-764.

[2] Ekström, E. And Wanntorp, H. (2009). Optimal stopping of a Brownian bridge. J. Appl. Prob. 46, $170-180$.

[3] Ethier, S. N. (2010). The Doctrine of Chances. Springer, Berlin.

[4] Ethier, S. N. AND LeVIN, D. A. (2005). On the fundamental theorem of card counting, with application to the game of trente et quarante. Adv. Appl. Prob. 37, 90-107.

[5] Gottlieb, G. (1985). An analytic derivation of blackjack win rates. Operat. Res. 33, 971-988. 
[6] Griffin, P. (1976). The rate of gain in player expectation for card games characterized by sampling without replacement and an evaluation of card counting systems. In Gambling and Society: Interdisciplinary Studies on the Subject of Gambling, ed. W. R. Eadington, Charles C. Thomas Publishing, Springfield, IL, pp. 429-442.

[7] Liese, F. AND Miescke, K.-J. (2008). Statistical Decision Theory. Springer, New York.

[8] McLeish, D. L. (1974). Dependent central limit theorems and invariance principles. Ann. Prob. 2, 620-628.

[9] Milbrodt, H. (1987). An invariance principle for $U$-statistics in simple random sampling without replacement. Metrika 34, 195-200.

[10] Thorp, E. O. (1962). Beat the Dealer: A Winning Strategy for the Game of Twenty-One. Random House, New York.

[11] Thorp, E. O. And Walden, W. E. (1973). The fundamental theorem of card counting with applications to trente-et-quarante and baccarat. Internat. J. Game Theory 2, 109-119. 